\title{
Un commis des trésoriers généraux de la marine à Québec. Nicolas Lanoullier de Boisclerc
}

\section{John Keyes}

Volume 32, numéro 2, septembre 1978

URI : https://id.erudit.org/iderudit/303689ar

DOI : https://doi.org/10.7202/303689ar

Aller au sommaire du numéro

Éditeur(s)

Institut d'histoire de l'Amérique française

ISSN

0035-2357 (imprimé)

1492-1383 (numérique)

Découvrir la revue

Citer cet article

Keyes, J. (1978). Un commis des trésoriers généraux de la marine à Québec. Nicolas Lanoullier de Boisclerc. Revue d'histoire de l'Amérique française, 32(2), 181-202. https://doi.org/10.7202/303689ar d'utilisation que vous pouvez consulter en ligne. 


\section{UN COMMIS DES TRÉSORIERS GÉNÉRAUX DE LA MARINE À QUÉBEC NICOLAS LANOULLIER DE BOISCLERC}

JOHN KEYES

Université Laval

\section{Introduction}

Le poste de commis des trésoriers généraux de la Marine à Québec, sous le Régime français, est encore mal connu. Pourtant, il s'agissait d'une fonction-clé, au centre du système financier de la Nouvelle-France. Dans L'administration de la Nouvelle-France (1929), Gustave Lanctôt l'a mal compris et puis Guy Frégault l'a à peine touché dans son «Essai sur les finances canadiennes (17001750)» (1959), et son volume sur La civilisation de la NouvelleFrance (1969). Plus récemment traitée par J.F. Bosher dans le cadre des finances françaises, la charge reste mal intégrée dans l'histoire de la colonie ${ }^{1}$. En reconstituant la carrière d'un des responsables de cette charge, cette étude se propose d'éclairer un peu le caractère, les fonctions et les problèmes de la gérance de la caisse de la Marine en Nouvelle-France.

Nicolas Lanoullier de Boisclerc fut un personnage très en vue dans les milieux administratifs, politiques et commerciaux de l'époque. Né vers 1679, fils de Jean de Lanoullier, bourgeois de Paris, et parent de la nourrice du Roi, il fut avocat au parlement avant d'aller en Nouvelle-France en 1712. Initialement envoyé à Québec comme agent de la Compagnie du Castor par Louis-François

1 Lanctôt, G. L'administration de la Nouvelle-France (1929); Guy Frégault, La civilisation de la Nouvelle-France, 1713-1744 (1969) et «Essai sur les finances canadiennes (1700-1750)» RHAF, XII \& XIII (1958-1959); J.F. Bosher, French Finances, 1770-1795: from business to bureaucracy, (1970) et «Governement and Private Interests in New France,» Canadian History Before Confederation, (1972), 111-124. 
Aubert, Jean-Baptiste Néret et Jean-Baptiste Gayot ${ }^{2}$, détenteurs du monopole du castor, ses affaires prirent passablement d'ampleur par la suite. En plus de s'occuper du castor pour le compte de la Compagnie du Castor de 1712 à 1717, puis pour celui de la Compagnie des Indes de 1726 à $1731^{3}$, il bénéficia de deux privilèges royaux: un pour faire bâtir les «moulins sur bateaux» sur le fleuve Saint-Laurent et l'autre pour le service postal dans la colonie ${ }^{4}$.

À la mort de Charles de Monseignat en 1718, il a vainement demandé la commission du Domaine ${ }^{5}$. Représentant des trésoriers généraux à Québec de 1720 à 1730, il devint ensuite contrôleur du Domaine d'Occident ${ }^{6}$. En 1733, 1734 et en 1736, il fit une demande pour obtenir la ferme de Tadoussac et, selon la correspondance de la colonie, le ministre préféra sa candidature aux autres. Néanmoins, l'intendant Gilles Hocquart recommanda la continuation de la régie de la ferme de Tadoussac sous le Domaine du Roi à laquelle elle avait été réunie en $1732^{7}$. Les propriétés foncières de Lanoullier en Nouvelle-France comprenaient la seigneurie du lac Métis achetée de Louise le Gardeur en $1725^{8}$, et un arrière-fief dans la seigneurie des Plaines qu'il perdit en 1749 faute d'y avoir établi des habitants'. Nommé au Conseil Supérieur en 1722, Lanoullier servit brièvement comme procureur général intérimaire après la mort de Mathieu-Benoît

2 Vaudreuil à Pontchartrain, 6 novembre 1712, $\mathrm{AC}, \mathrm{C} 11 \mathrm{~A}, 33$ : 68; Bégon à Pontchartrain, 12 novembre 1712, C11A, 33: 141 .

3 Beauharnois et Dupuy à Maurepas, 20 octobre 1726, C11A, 48: 63-63v; "Syndics et Directeurs de la Compagnie des Indes à M. Hocquart», 23 mai 1731, C11A, 56: 228-228v.

4 Tous les deux sont restés lettres mortes. Vaudreuil et Bégon au Conseil de la Marine, 10 novembre 1721, C11A, 44: 126-127; Placet de Lanoullier, ibid., 128129v; Vaudreuil et Bégon, 2 novembre 1724, C11A, 46: 40v; Ordonnance du 27 janvier 1721, Inventaire des ordonnances des intendants de la Nouvelle-France, par P.G. Roy (Beauceville, 1919), I: 194.

5 Hocquart à Maurepas, 8 octobre 1733, C11A, 60: 62.

6 «Nicolas Lanoullier. À Messieurs les Fermiers généraux des domaines de France. Ferme de Tadoussac. Droits de Domaine,» (C11A, 50: 409-412 (faussement daté 1728).

7 Hocquart, 8 octobre 1733, C11A, 60:65-65v; Lanoullier à Maurepas, avril 1733, C11A, 61: 269; Lanoullier, 10 octobre 1734, C11A, 62: 300-301v. En 1735 Hocquart recommanda François-Étienne Cugnet comme fermier des postes de Tadoussac, sans aucune mention de Lanoullier. Beauharnois et Hocquart, 15 octobre 1735, C11A, 63: 110; Hocquart, 15 octobre 1735, C11A, 64: 109-116. Lanoullier, 9 octobre 1736, C11A, 66: 119-120v.

8 Archives de la province de Québec, Inventaire des concessions en fief et seigneurie (Beauceville, 1928), 63-64.

9 Ibid., 236. 
Collet en $1727^{10}$ et fut désigné garde des sceaux du conseil en $1735^{11}$. Pendant les années 1740, le gouverneur Beauharnois lui accorda des gratifications annuelles de $\mathbf{3 0 0 0}$ livres sur l'exploitation des congés de traite $^{12}$. En outre, durant toutes ces années, il poursuivit un commerce considérable des marchandises françaises vendues à Québec ${ }^{13}$.

Ses liens familiaux lui furent avantageux, tant dans la colonie qu'en France. Ainsi son mémoire demandant la charge de directeur du Domaine en 1718 fut présenté par son parent, "Madame la Nourrice du Roi ${ }^{14}$. Plus tard, dans le cadre de ses fonctions de commis des trésoriers généraux son père plaida en sa faveur auprès du ministère de la Marine ${ }^{15}$. Dans la colonie, son demi-frère, JeanEustache Lanoullier de Boisclerc, servit comme contrôleur de la Marine de 1719 à 1729, années pendant lesquelles Lanoullier fit office de commis des trésoriers ${ }^{16}$. Un troisième frère Lanoullier, Paul-Antoine-François des Granges, était écrivain du Roi à Québec ${ }^{17}$. De plus, le premier mariage de Nicolas Lanoullier à Jeanne-Catherine André de Leigne, l'a lié à Pierre André de Leigne, lieutenant général de la Prévôté et aussi à la famille Rouville, qui comprenait des seigneurs et des commandants de postes ${ }^{18}$.

10 «Lettres de provisions au Sieur Lanoullier pour l'office de conseiller au Conseil Supérieur," 10 février 1722, Inventaire des jugements et délibérations du Conseil Supérieur de la Nouvelle-France de 1717 à 1760 (Beauceville, 1935), VI: 260-261. Voir aussi, Vaudreuil et Bégon, 17 octobre 1722, C11A, 44: 300v; Beauharnois, 27 octobre 1727, C11A, 49: 92 (Beauharnois remarqua l'alliance entre l'intendant Dupuy et Lanoullier au conseil en 1727).

11 "Lettres de provisions au Sieur Lanoullier pour l'office de garde des sceaux du Conseil Supérieur," 21 mai 1735, Inventaire... du Conseil Supérieur, VI: 261.

12 Lanoullier au Roi (sans date, mais probablement de 1748), C11A, 61: 272273.

13 Maurepas à Beauharnois et Dupuy, 13 mai 1727, AC, B, 50: 528; Hocquart, 14 octobre 1730, C11A, 53: 24-25; Hocquart, 8 octobre 1733, C11A, 60 : 66.

14 «A Messieurs les Fermiers," C11A, 50: 412. Elle fit aussi des recommandations en faveur de Lanoullier pendant les années 1740, C11A, 61: 272.

15 Maurepas à Hocquart, 10 avril 1731, C11A, 56: 25-25v.

16 "Mémoire du Roi à Messieurs le Marquis de Vaudreuil et Bégon,» 23 mai 1719, B, 41: 525v; Vaudreuil et Bégon, 26 octobre 1719, C11A, 40: 48v; Hocquart, 25 octobre 1729, C11A, 51: 267-267v; Maurepas à Hocquart, 22 mai 1729, B, 53: 556v. Les deux frères ont été confondus de temps en temps par certains historiens. Voir, par exemple, Adam Shortt, Documents relatifs à la monnaie (Ottawa, 1925), 602.

17 P.-G. Roy, La famille Lanoullier (Lévis, 1935), 24.

18 Ibid., 16; Cameron Nish, "La bourgeoisie et les mariages,» RHAF, XIX (mars 1966): 597. 
Dans le cadre de cette recherche, c'est en tant que commis des trésoriers généraux de la Marine que Nicolas Lanoullier de Boisclerc nous intéresse le plus. Cependant, cette fonction ne saurait être isolée du contexte politique et économique de l'époque, car celui-ci eut une influence considérable dans l'exercice de cette fonction. Lanoullier fut nommé à cette charge en 1720 , probablement à la suite de pressions de sa famille à Paris, quoique les détails de sa nomination restent inconnus, la correpondance officielle de l'époque n'en faisant point mention. Il y est demeuré jusqu'à ce qu'un vide dans sa caisse soit découvert en 1730 , ce qui entraîna sa destitution.

\section{Les trésoriers généraux de la Marine}

Comprendre le rôle du commis des trésoriers généraux de la Marine dans la vie politique de la Nouvelle-France exige une connaissance du poste de trésorier général lui-même en France. Contrairement à la situation àctuelle, où les finances de l'État sont gérées par un «receveur général» qui est directement responsable au gouvernement, l'Ancien Régime établissait une nette distinction entre la fonction d'ordonnateur et celle de comptable. La deuxième relevait d'un trésorier qui était indépendant des ministres d'État ${ }^{19}$. Depuis l'époque de Jean-Baptiste Colbert, qui avait régularisé ce système pendant son ministère, le trésorier qui achetait son office était un simple dépositaire, sans aucun pouvoir d'ordonner la dépense des fonds dont il avait la charge. Il devait effectuer des déboursés uniquement sur les ordres des administrateurs chargés de ce pouvoir, soit les ministres, les intendants et les commissaires-ordonnateurs. Un tel système est un reflet assez fidèle de l'Ancien Régime, caractérisé, comme on le sait, par la vénalité des charges et une organisation financière mal intégrée. Les trésoriers, qui alternaient d'année en année ${ }^{20}$, étaient davantage des banquiers que des fonctionnaires royaux. Ils devaient répondre des deniers royaux déposés dans leurs caisses; mais dès leur entrée jusqu'à celui de leur sortie, ces derniers

19 Henri Legohérel, Les trésoriers généraux de la Marine (Paris 1963); J.F. Bosher, French Finances, 1770-1795; from business to bureaucracy (Cambridge, 1970), et "Governement and Private Interests in New France," Canadian History Before Confederation, J.M. Bumsted, ed., (Georgetown, Ontario, 1972), 111-124.

${ }_{20}$ D'habitude il y avait deux trésoriers à la fois, un qui était en exercice et un autre qui travaillait à régler ses comptes pour son exercice de l'année précédente. Pendant les années de la commission de Lanoullier, de 1720 à 1730, il y avait trois trésoriers qui étaient, chacun, en exercice chaque troisième année. (Gaudion, Moufle de la Thuilerie, et De Selle) Legohérel, 324-325. 
échappaient totalement au contrôle royal. Souvent, seule la mort d'un titulaire du poste, en nécessitant un inventaire des comptes, révélait des débits ou des malversations ${ }^{21}$.

Dans les principaux ports de France et dans les colonies, les trésoriers étaient représentés par des commis, distincts des officiers royaux établis sur les lieux. Au Canada le commis ne fut jamais investi d'un brevet ou d'une commission du Roi comme le furent les deux autres officiers qui avaient un droit de surveillance sur la caisse de la Marine - le commissaire et le contrôleur ${ }^{22}$. De plus, les gouverneurs et les intendants l'ont toujours désigné comme «commis du trésorier» ou "des trésoriers» mais jamais comme officier du $\mathrm{Roi}^{23}$. La correspondance officielle échangée entre l'administration coloniale et le ministère de la Marine révèle parfois assez clairement à qui le commis était responsable. Ainsi, en 1720, le Conseil de la Marine informa l'intendant Michel Bégon que «les Trésoriers Généraux de la Marine ont représenté que leur commis en Canada se plaint qu'il a beaucoup de peine à retirer en bonne forme les décharges des dépenses qui se font à Montréal» ${ }^{24}$.

De fait, le droit de nommer ces commis appartenait nettement aux trésoriers généraux et c'est seulement dans des circonstances exceptionnelles que l'intendant a procédé à des nominations. En 1730, par exemple, suite à la découverte d'un vide dans la caisse de Lanoullier, Hocquart confia les fonds de la Marine à un de ses secrétaires, le sieur Nicolas Bauve. L'année suivante, les trésoriers voulurent en faire leur commis attitré, mais Bauve refusa la commission ${ }^{25}$.

La différence entre le commis des trésoriers et les officiers royaux dans la colonie se vérifie aussi dans la source de ses appoin-

21 Bosher donne l'exemple de Louis Barthelemy Mouffle de Geoville, trésorier de 1744 à 1762, qui décéda sans avoir régler ses comptes pour 1756, 1758, 1760 et 1762 , dont on trouva un débit de 1659000 livres. Bosher, « Government and Private Interst », 118. Voir aussi, Bosher, French Finances, 107-110.

22 Vaudreuil et Bégon, 26 octobre 1719, C11A, 40: 48v. Le commissaire fut le représentant de l'intendant à Montréal et, dans les instances où la colonie se trouvait sans intendant, ce fut lui qui remplissait les fonctions de l'intendance. Le contrôleur s'occupait de plus près de la comptabilité de la Marine.

${ }_{23}$ Vaudreuil et Bégon, 12 novembre 1712, C11A, 33: 28; Vaudreuil et Bégon 26 octobre 1720, C11A, 42: 63v.

24 Conseil de la Marine à Bégon, 20 avril 1720, C11A, 41: 258.

25 Maurepas à Hocquart, 8 mai 1731, C11A, 56: 30; Hocquart, 18 octobre 1731, C11A, 55: 246; Hocquart, 5 octobre 1732, C11A, 58: 253v. 
tements. À l'époque de Lanoullier, ces appointements qui se montaient à 1200 livres étaient payés par les trésoriers généraux alors que les appointements du commissaire et du contrôleur de la Marine étaient inclus dans l'État du Roi ${ }^{26}$. Quand en 1725, Lanoullier se plaignit de l'insuffisance de son traitement, Bégon et Longueuil représentèrent dans une lettre commune qu'il serait juste qu'il soit doublé par les trésoriers ${ }^{27}$. L'intendant Gilles Hocquart revint à la charge plusieurs fois à ce sujet. Finalement, pour soulager quelque peu le commis, Hocquart ordonna le paiement de 600 livres au compte du Roi, mais il a bien souligné que «sa Majesté ne doit point être tenue de ces sortes de dépenses ${ }^{28}$.

\section{Fonctions du commis à Québec}

Les fonctions du commis des trésoriers généraux étaient essentiellement celles d'un caissier. Chaque année, en fin d'été, le vaisseau du Roi arrivait à Québec avec l'État du Roi préparé par le ministre suivant le projet des dépenses envoyé l'automne précédent par l'intendant. Par le même vaisseau, les trésoriers de la Marine envoyaient à leur commis les fonds requis pour payer ces dépenses ${ }^{29}$. Habituellement une partie de ces fonds était remise en deniers comptants et une autre en marchandise pour le magasin du Roi. Le commis recevait aussi des acquits pour les déboursés déjà faits en France, normalement pour payer des appointements et des ordres relatifs aux lettres de change à faire tirer sur le trésorier en exercice ${ }^{30}$. Ces fonds et documents étaient déposés directement entre les mains du commis et gardés en caisse chez lui ${ }^{31}$. D'autres sommes y étaient

26 Longueuil et Bégon, 31 octobre 1725, C11A, 47: 118v; « Projet des dépenses à faire par le Roi en la Nouvelle-France pour le service de sa Majesté,» (sans date), C11A, 50: 305-315; Hocquart, 8 octobre 1733, C11A, 60: 67-67v.

27 Longueuil et Bégon, 31 octobre 1725, C11A, 47: 118v.

28 Hocquart, 5 octobre 1732, C11A, 58: 242-252v. Voir aussi, Hocquart, 16 octobre 1730, C11A, 53: 55v-56; 18 octobre, C11A, 55: 245v. Les 600 livres furent pour l'entretien d'un sous-commis à Montréal.

29 Faute de banque centrale, les trésoriers généraux eux-mêmes ont reçu leurs propres fonds des diverses caisses centrales et provinciales selon les assignations du Trésor Royal. Le fonctionnement de ce système, particulièrement pour le dixhuitième siècle, fut marqué d'une «absence totale de toute direction cohérente: les fonds, quand il y en a, proviennent des premières caisses où l'on trouve quelque chose». Legohérel, 274. Voir aussi 109-114; et Bosher, French Finances, 67 \& 92.

30 C11A, 44: 178-182.

31 Quelques fonds étaient gardés séparément comme ceux provenant de la vente des marchandises du Roi, mais toujours chez le commis. 
parfois aussi versées: telles les impositions prélevées pour la construction de l'enceinte de Montréal, les rentes dues au Roi, etc ${ }^{32}$ Avec ces sommes le commis commençait immédiatement à pourvoir aux dépenses sur les ordres de l'intendant ${ }^{33}$.

Les fonds remis aux commis ne constituaient pas la seule ressource financière de l'administration de la Nouvelle-France. Il existait d'autres caisses dans la colonie, comme celle du Domaine d'Occident, mais la plus forte partie des dépenses dépendait de la caisse du commis des trésoriers généraux ${ }^{34}$. Ce commis payait la solde de l'État-major et des soldats des troupes de la marine, les appointements des administrateurs, les gratifications ordonnées par le Roi, le coût des ouvrages de la Marine et celui des vivres et des hardes ${ }^{35}$. Les dépenses nécessaires pour l'équipage des navires royaux venus en Canada et pour les produits de la colonie - le bois, le goudron et le chanvre - envoyés aux ports de France, étaient pourvues par le commis, quoique d'habitude ces déboursés aient été remboursés l'année suivante ${ }^{36}$.

Les comptes du commis étaient sujets à des vérifications périodiques, normalement en septembre ou en octobre, par l'intendant d'abord, puis par le commissaire et le contrôleur de la Marine. Chaque année, l'intendant devait envoyer au ministère de la Marine un compte rendu du travail officiel du commis, c'est-à-dire de ses déboursés pour divers services, appointements et soldes, des lettres de change qu'il avait tirées, et, sous le régime de la monnaie de cartes, un bordereau de celles qui avaient été retirées chaque année ${ }^{37}$. De plus, des procès-verbaux des espèces trouvées dans

32 Vaudreuil et Bégon, 2 novembre 1724, C11A, 46: 44v; Collet, procureur général, 5 novembre 1712, C11A, 33: 190-193v; Vaudreuil et Bégon, 14 octobre 1716, C11A, 36: 14, (ils demandèrent la vente du quart du castor en Canada pour augmenter la caisse de la colonie).

33 Bégon, 26 octobre 1722, C11A, 44: 387.

34 Bégon, 14 octobre 1723, C11A, 45: 234v-235; Dupuy, 14 octobre 1726, C11A, 48: 263-263v (il parle des caisses de Lanoullier, de Cugnet, directeur du Domaine, et de Boisclerc, receveur des invalides de la Marine).

35 Vaudreuil et Bégon, 26 octobre $1720, \mathrm{C} 11 \mathrm{~A}, 42: 78-78 \mathrm{v} ; 8$ octobre 1721 , C11A, 44: 36v; 4 novembre 1721, C11A, 44; 100-100v; Vaudreuil, 11 novembre 1721, C11A, 44; 179v; «Projet des dépenses", C11A, 50: 305-315.

36 Beauharnois et Dupuy, 14 octobre 1726, C11A, 48: 7; "État des lettres tirées», 23 octobre 1723, C11A, 46: 292a; Hocquart, 15 janvier 1731, C11A, 55: 5v.

37 Vaudreuil et Bégon, 12 novembre 1712, C11A, 33: 22; "État général des Lettres de change tirées», C11A, 38:80-83. 
les diverses caisses du commis étaient dressés et signés par les trois officiers en présence du commis ${ }^{38}$. Après 1729 , on retrouve la signature du contrôleur de la Marine à côté de celle de l'intendant sur les états des lettres de change. De plus, le contrôleur devait procéder aux recouvrements nécessaires des anciens commis ou de leurs héritiers après l'arrêté final des comptes ${ }^{39}$.

Le travail des commis en Canada était rendu difficile par les aléas des finances françaises et par certains problèmes propres à la colonie. Ils subirent d'abord constamment le contrecoup des problèmes financiers de l'époque et leurs comptes en furent dérangés. Entre 1702 et 1742 , les projets de dépenses envoyés par l'intendant furent presque toujours réduits par le ministre malgré les supplications régulières des intendants de n'y rien retrancher ${ }^{40}$. Face à cette pénurie presque constante de fonds, la colonie devait excéder les prévisions des états du Roi et rejeter une partie des dépenses d'une année sur le compte de l'année suivante, en faisant tirer par le commis des lettres de change sur le trésorier en exercice cette année-là ${ }^{41}$. Ainsi, les comptes et les fonds des années différentes furent confondus les uns avec les autres. En 1727, l'intendant Dupuy prépara un dépouillement des comptes des années 1711 à 1727 qui fit voir que ces excédents de dépenses s'étaient accumulés d'année en année "au point que l'année 1728 se trouve presque consommée ${ }^{42}$.

En plus de ce manque de fonds, la colonie était également aux prises avec une pénurie d'espèces qui soulevait d'autres problèmes dans la gestion de la caisse de la Marine. La monnaie de cartes, dont la première émission fut lancée par l'intendant Jacques de Meulles en 1685, avait été l'une des principales mesures pour pallier à cette disette. Ces cartes furent entièrement retirées sur les ordres du Conseil de la Marine en 1719, mais la colonie dut revenir à ce système en 1729 pour soutenir le commerce interne ${ }^{43}$. Comme caissier de la colonie, les commis des trésoriers signaient ces cartes

38 Procès-verbal des espèces, 10 octobre 1724, C11A, 47. 54-54v; Procèsverbal des espèces, 26 août 1725, C11A, 47: 56-57.

39 Poursuite contre la Dame veuve Duplessis, par Jean-Eustache Lanoullier de Boisclerc, 1719, C11A, 36: 157-158v.

40 Voir Frégault, «Essai sur les finances canadiennes», 318.

41 «État des lettres de change tirées», 10 octobre 1725, C11A, 47: 45-53.

42 Beauharnois et Dupuy, 20 octobre 1727, C11A, 49: 45-47v.

43 Beauharnois et Hocquart, 25 octobre 1730, C11A, 54 : 298-301. 
avec l'intendant et le gouverneur; le maniement de ce système monétaire s'avéra une tâche lourde et difficile pour eux. Après le retrait de toute la monnaie de cartes en 1719, le gouverneur Vaudreuil et l'intendant Bégon diront, au sujet du commis décédé, Jean Petit, que «ce grand travail l'a tellement épuisé qu'il a toujours été malade de ce temps-là jusqu'à sa mort ${ }^{44}$.

Enfin, la tenue des comptes se compliquait, du seul fait de la distance qui séparait la colonie de la métropole. Il s'est avéré presque impossible de réunir les pièces justificatives nécessaires pour arrêter des comptes, surtout à cause des paiements faits en France et mis au compte de la colonie et des lettres de change tirées en Canada et payables en France sur un ou plusieurs exercices ${ }^{45}$. En conséquence, les comptes des commis pouvaient demeurer en suspens pendant des années. Le problème devenait encore plus aigu quand la comptabilité des trésoriers généraux en France ne coïncidait pas avec celle de leur commis à Québec. On trouve, par exemple, dans la correspondance envoyée au ministre en 1725 , un «État des fonds à remettre en Canada l'année prochaine 1726». À cette occasion, l'intendant Bégon demandait la remise de 12380 livres pour les présents faits aux sauvages en 1716, «laquelle a été retenue sur celle de 318393 livres destinée pour les dépenses de la colonie de ladite année. Le Conseil a ordonné qu'elle serait remplacée par M. Gaudion (trésorier général), ce qui n'a pas été fait ». Il réclamait aussi 56636 livres pour les dépenses des vaisseaux du Roi entre les années 1716 à $1723^{46}$. En 1729 , le trésorier de Selle fut enjoint de remettre 19562 livres pour les exercices de 1721 et 1727 mais, après avoir consulté ses comptes, il représenta que c'était son commis, Lanoullier, qui lui devait cette somme. François Clairambault d'Aigremont, commissaire de la Marine remplissant les fonctions d'intendant, répliqua que «bien loin que le commis dût cette somme à $\mathbf{M}$. de Selle» le trésorier devait à son commis 2336 livres pour le compte de 1722 et 8108 livres pour celui de $1725^{47}$. Jusqu'où pouvait aller la confusion fut révélé en 1731 lorsque Hocquart se plaignit des trésoriers généraux qui n'avaient pas encore remboursé à Lanoullier tous les fonds dépensés en 1716 pour les vivres envoyés à l'Ile Royale ${ }^{48}$.

\footnotetext{
44 Vaudreuil et Bégon, 6 novembre 1720, C11A, 42: 150-152.

45 Dupuy, 20 octobre 1727, C11A, 49: 322v.

46 C11A, 47: 41-42.

47 B, 53: 495-495v.

48 Hocquart, 15 octobre 1731, C11A, 55: 162.
} 
Mis à part leurs fonctions financières, les commis des trésoriers en Canada jouaient un assez grand rôle dans la vie politique de la colonie, tant dans l'intimité de l'intendant qu'au sein du Conseil Supérieur. Les deux personnes qui se partagèrent les fonctions de commis au début du siècle - Georges-Regnard Duplessis et Jean Petit - avaient succédé à l'oncle de ce dernier, Jacques Petit de Verneuil qui était décédé en $1699^{49}$. Petit l'aîné avait agi comme simple comptable, tel qu'envisagé par Colbert, et n'avait pas participé aux affaires politiques. Mais son neveu fut nommé conseiller au Conseil Supérieur en 1718, et Duplessis cumula en plus les charges de receveur des droits de l'amirauté et de directeur de la Compagnie de la Colonie ${ }^{50}$. Dans la fameuse série de querelles entre l'intendant Claude-Thomas Dupuy et le gouverneur Beauharnois, Lanoullier, en qualité de conseiller et, pour un court temps, procureur général du Conseil Supérieur, fut un allié important de l'intendant ${ }^{51}$. Le commis intérimaire de 1730 à 1732, Nicolas Bauve, et l'éventuel remplaçant officiel, Thomas-Jacques Taschereau, vinrent tous deux des bureaux de l'intendance. Bauve combina brièvement les fonctions de secrétaire de Hocquart avec celles de la caisse de la Marine et Taschereau devint secrétaire de l'intendant Dupuy. Les deux successeurs permanents de Lanoullier, Taschereau, de 1732 jusqu'à son décès en 1749, et Jacques Imbert, de 1749 à 1758, furent aussi nommés au Conseil ${ }^{52}$. À cette époque donc, les commis des trésoriers, soit par leurs connaissances juridiques, soit par leurs fortunes personnelles, figuraient parmi ces «très peu de personnes dans cette colonie capables de remplir les places de judicature ${ }_{\gg}{ }^{53}$. Venant s'ajouter à leur rôle financier, ces fonctions politiques firent des commis des personnages importants dans l'administration de la colonie.

49 P.-G. Roy, La ville de Québec (Québec, 1930), 473. De 1710 à 1719, Duplessis et Petit ont partagé le poste en alternance d'année en année. Collet, procureur général, à Pontchartrain, 5 novembre 1712, C11A, 33: 192v; Bégon, 12 novembre 1712, C11A, 33: 145.

so Collet, ibid.

51 Beauharnois, 27 octobre 1727, C11A, 49: 92. Voir aussi Dale S. Standen, Charles, Marquis de Beauharnais de la Boische, Governor General of New France, 1726-1747 (University of Toronto, Thèse de Ph.D., 1975). Lanoullier s'est retiré de la situation avant la révocation de Dupuy.

52 Roy, La ville de Québec, 473-474 et 271-272.

53 Beauharnois et Hocquart, 13 novembre 1729, C11A, 51: 115-116 (Ils se sont plaints des émoluments des places de judicature). 


\section{L'exercice de Lanoullier de Boisclerc}

La carrière de Lanoullier de 1720 à 1730 illustre bien les difficultés du poste qu'il occupait et plus spécifiquement la situation embarrassée des finances de la colonie. La disette d'espèces fut un problème auquel Lanoullier dut s'attaquer dès son entrée en fonction; les difficultés rencontrées dans l'arrêt des comptes entraînèrent de grands délais dans l'accomplissement de sa tâche. La nature strictement indépendante de la gestion de sa caisse et la poursuite de ses propres affaires commerciales firent planer de graves soupçons sur lui, tant dans la colonie que chez les trésoriers en France. Enfin, ses relations avec les intendants Bégon et Dupuy et autres officiers de la colonie favorisèrent un système d'avances qui n'était pas tout à fait inconnu à l'époque mais qui finalement causa sa perte.

La première crise éclata dès l'année qui suivit sa nomination. Elle concernait la dépense des fonds envoyés en argent par le vaisseau du Roi en 1721. Le gouverneur Philippe de Rigaud de Vaudreuil se plaignit qu'en dépit de l'envoi de 188000 livres en argent, somme comprenant les fonds ordonnés pour 1721 et 60000 livres à compter de l'année suivante spécifiquement affectées à la solde des troupes et aux appointements des officiers, Lanoullier avait fermé sa caisse à peine un mois après l'arrivée du vaisseau. Au dire du gouverneur, le commis avait ainsi privé une partie des officiers de leurs appointements pour les mois de juillet, août et septembre. Au lieu d'argent, il leur avait offert des marchandises. Quand le gouverneur exigea des explications, Lanoullier répondit que cela ne le concernait pas, car il n'effectuait des paiements que sur l'ordre de l'intendant et tant qu'il y avait des fonds ${ }^{54}$.

L'année suivante, Bégon se porta à la défense de Lanoullier. N'ayant pas eu le temps de vérifier lui-même les livres de caisse du commis, il l'avait simplement mis au courant des plaintes qu'on portait contre lui. Lanoullier l'avait assuré

...que tous les officiers ont été payés en espèces à l'arrivée du vaisseau du Roi l'année dernière de leurs appointements et solde des compagnies des 9 premiers mois à l'exception de cinq de la garnison de Montréal qui n'étaient point alors descendus ici, lesquels ont été payés par son commis en ladite ville au mois de novembre suivant... s'il y en avait quelqu'un qui n'eût pas été

54 Vaudreuil, 11 novembre 1721, C11A, 44: 178-182. 
payé des 9 premiers mois il ne serait pas resté dans le silence et j'en aurais été averti. Aucun ne m'en a parlé ni écrit ${ }^{55}$.

Selon Bégon, c'était le désir des officiers d'être payés en octobre 1721 pour les trois derniers mois de l'année qui avait donné lieu aux plaintes et Lanoullier ne disposait pas des fonds nécessaires pour se rendre à cette demande.

Nonobstant ces explications, les plaintes concernant le traitement des troupes de la Marine furent renouvelées presque annuellement par la suite. Tous les ans, avec l'État du Roi, la même somme de 60000 livres, acompte de l'année suivante, était envoyée avec les mêmes instructions: «à payer la solde des soldats de leurs appointements et de quelques officiers les plus en necessité... et ce fonds ne sera pas employé à d'autres usages " 56 . Néanmoins, en octobre 1722, Vaudreuil écrit que le commis n'a plus que 19000 ou 20000 livres de cette somme en caisse ${ }^{57}$. Quatre ans plus tard, en 1726, Lanoullier ne put apparemment payer tous les appointemments. Selon l'intendant Dupuy, cependant, cela était dû au manque d'espèces.

La raison pour laquelle il ne fut payé l'année précédente aux troupes que les six premiers mois, fut que les officiers qui reçoivent leurs appointements et qui exploitent leurs compagnies ne voulurent point être payés en lettres de change, qu'il était cependant le seul paiement qui pût leur être fait attendu que sur 149000 livres qui fait le paiement ordinaire des troupes, il n'était venu d'argent comptant dans la colonie que la somme de 60000 livres ${ }^{58}$.

Et Dupuy d'ajouter que Lanoullier s'était ainsi justifié du reproche d'avoir obligé les officiers d'accepter des marchandises. D'après l'intendant, il n'avait eu recours à cet expédient qu'à deux occasions, pour des militaires qui exigeaient d'être payés d'avance pour leurs appointements et qui avaient accepté la valeur en marchandise ${ }^{59}$. Mais le ministre n'en éprouvait pas moins des doutes à l'égard du commis et il donna ordre aux intendants d'exercer une étroite sur-

5s Bégon, 26 octobre 1722, C11A, 44: 384-384v. Voir aussi, Conseil à Bégon, 5 mai 1722, B, 45: 761-763.

56 Beauharnois et Dupuy, 12 octobre 1726, C11A, 48: 3v; Dupuy, 21 octobre 1726, C11A, 48: 292.

57 Vaudreuil, 20 octobre 1722, C11A, 44: 353-354v.

58 Dupuy, 27 octobre 1727, C11A, 49: 416-416v.

59 Ibid., 417v-418. 
veillance à son égard, particulièrement en ce qui avait trait au paiement des troupes ${ }^{60}$.

Cette attitude méfiante s'explique bien. Déjà suspecté d'irrégularités au chapitre du paiement des troupes, Lanoullier était aussi soupçonné de détourner des fonds royaux pour faire son propre commerce, ce qui était nettement plus grave. La diminution du nombre d'espèces envoyées dans la colonie était imputable, selon Maurepas, au grand nombre de lettres de change tirées par Lanoullier; quelques-unes, faites à l'ordre de son père, marchand de Paris, montaient à 40000 livres.

Il est même à craindre que le commis des Trésoriers n'abuse de cette facilité, et qu'il ne se fasse par cette voie des fonds pour faire envoyer des marchandises de France pour les revendre dans la colonie, peut être en partie aux officiers pour leurs appointements. Mon soupçon sur cela n'est point sans quelque fondement, puisque le père de ce commis a porté à $\mathbf{M}$. Gaudion pour plus de $40 \mathrm{~m}$ livres de lettres tirées l'année dernière dont il a reçu le paiement ${ }^{61}$.

Cette année-là, 1726, Dupuy précisa au ministre qu'il avait surveillé le commis de près et avait continué de viser les lettres de change pour qu'il n'en tirât aucune sans les ordres et les quittances des parties prenantes ${ }^{62}$. Dans une lettre commune de 1727 , le gouverneur et l'intendant qualifièrent de «sourdes", «indéterminées» et somme toute non justifiées les plaintes contre Lanoullier ${ }^{63}$.

Même si ces accusations ne furent jamais prouvées, il n'en demeure pas moins que Lanoullier a entretenu un négoce considérable avec son père et d'autres commerçants français. En 1730, au moment où s'effectuait la vérification de ses comptes, il demanda à Hocquart la délivrance des marchandises appartenant à son père et au «Sieur Le moine marchand de Rouen». L'intendant la lui refusa, prétextant la condition douteuse de ses affaires ${ }^{64}$. L'ampleur de son commerce est bien illustrée aussi par le montant des dettes mention-

60 Maurepas à Dupuy, 14 mai 1726, B, 49: 609-619; Dupuy, 20 octobre 1727, C11A, 49: 279-279v.

61 «Mémoire du Roi pour servir d'instruction à M. Dupuy», 14 mai 1727, B, 50: 528.

62 Dupuy, 21 octobre 1726, C11A, 48: 294v-295.

63 Beauharnois et Dupuy, 20 octobre 1727, C11A, 49: 45v-46v; Dupuy, 27 octobre 1727, C11A, 49: 417v.

64 Hocquart, 14 octobre 1730, C11A, 53: 24-24v. 
nées dans ses papiers au moment de l'inventaire en septembre 1730 . Il devait alors à Le Moine, de Rouen, 6594 livres; à Julien, marchand à Paris, 5898 livres; à Lanoullier père, de Paris, 26958 livres; à Andrien Cornet, marchand d'Amiens, 9786 livres; à Pascaud, de la Rochelle, 9040 livres; à Bougin, de la Rochelle, 6326 livres; et à Mariette, de Montauban, environ 5000 livres, pour un total approximatif de 70000 livres ${ }^{65}$.

Une autre affaire de Lanoullier qui donna lieu à des soupçons impliquait l'intendant Bégon. Le 10 novembre 1724, la monnaie fut dévaluée d'un tiers. Juste avant cette date, Lanoullier accepta de la veuve de l'ancien commis Jean Petit la somme de 17000 livres acompte de ce qui était dû au Roi. Cette somme servit alors au paiement des dettes de Bégon à la succession de Petit. Comme conséquence de ces transactions douteuses, le Roi subit une perte de 5000 livres. De plus, certains prétendirent que des particuliers avaient été informés de la diminution avant la date de l'ordonnance ${ }^{66}$.

De toutes les pratiques douteuses qui caractérisèrent le travail de Lanoullier, celle qui fut éventuellement responsable de sa destitution n'était pas tout à fait inhabituelle à l'époque. Il s'agissait du consentement d'avances aux officiers. À vrai dire, cette pratique n'était pas totalement irrégulière, car ces avances ne constituaient que des prêts faits par le commis avec des deniers royaux et pour le recouvrement desquels il était tenu personnellement responsable. Le ministère de la Marine était d'ailleurs au courant de cette pratique qui illustre fort bien le rôle ambivalent des trésoriers et de leurs commis, agissant parfois comme des agents royaux et parfois comme des banquiers quasi-privés. Par exemple, suite à une avance faite par Lanoullier à de Morville, officier dans les troupes, le ministre refusa la remise par le roi pour le compte de la veuve de Morville. Il expliqua que le Roi ne voulait pas «entrer dans la discussion des prêts que le sieur Lanoullier a fait aux officiers ${ }^{67}$. De même, à l'occasion de la saisie des effets de l'intendant Dupuy, le ministre Maurepas refusa de considérer les avances du commis Lanoullier dans le cadre de ses fonctions officielles. Maurepas écrivit alors à D'aigremont, commissaire de la Marine remplissant les fonctions de l'intendant:

65 C11A, 53: 262-269.

66 Maurepas à Dupuy, 14 mai 1726, B, 46: 668-670; Dupuy, 20 octobre 1726, C11A, 48: 278-282.

67 Maurepas à Beauharnois et Hocquart, 8 mai 1731, C11A, 56: 80-81. (Cependant, deux années avant, la moitié de la somme avancée, 530 livres, avait été remise à la veuve de Morville. B, 53: 468). 
La conduite que vous avez tenue avec M. Dupuy depuis son rappel est d'autant plus blâmable qu'outre qu'il a été traité avec indignité le Roi n'avait aucun intérêt dans la créance du Sieur Foucault ni celle du Sieur de la Nouillier qui leur sont personnelles ${ }^{68}$.

Les trois principaux créanciers de Dupuy étaient le commis Lanoullier, François Foucault, garde-magasin, et le sieur Walon, un associé de Lanoullier dans les affaires de la Compagnie des Indes. Malgré les remarques du ministre, le Conseil Supérieur donna raison à Lanoullier comme premier créancier pour le recouvrement de la somme de 11862 livres due par Dupuy sur la vente de ses effets ${ }^{69}$.

Ce ne fut peut-être pas tant le fait d'accorder des avances que la facilité avec laquelle il les accordait qui perdit Lanoullier. Ironiquement ce fut ce même Dupuy qui rapporta au ministre en 1727: «je ne le trouve que trop facile à avancer à l'officier, sur quoi i'ai même été obligé de le retenir quelques fois à cause de sa propre sûreté et des conséquences qui s'en seraient ensuivies. ${ }^{70}$ Les deux bénéficiaires principaux de ces avances furent les intendants Bégon, pour un total de 63859 livres, suite aux pertes considérables que lui avaient occassionnées deux incendies et le naufrage du navire «Le Chameau " ${ }^{71}$, et Dupuy pour les 11862 livres dont il a déjà été question. Même le commissaire de la Marine, Charles Clairambault D'aigremont, qui partageait la surveillance de Lanoullier avec l'intendant et le contrôleur, se retrouvait son débiteur dans le cadre de ce système d'avances ${ }^{72}$.

Un procès-verbal des recouvrements d'avances préparé par Lanoullier en 1731 à l'usage de Bauve, le commis intérimaire, fait voir quelque peu l'étendue du système. De «plusieurs officiers de Montréal» il avait recouvré 9909 livres. Parmi dix-huit autres, il y avait de Perigny qui devait 3 livres 10 sous; Honoré Michel,

68 Maurepas à D'Aigremont, 12 avril 1729, B, 53: 491-492; «Mémoire de ce qui s'est passé au sujet de la dette de Monsieur et de Madame Dupuy ", ler octobre 1730, C11A, 52: 267v; Hocquart, 25 octobre 1730, C11A, 53: 199.

69 Inventaire des jugements et délibérations du Conseil supérieur de la Nouvelle-France de 1717 à 1760, II, par P.-G. Roy (7 août 1730): 118; (14 août 1730); 119; (27 septembre 1730): 125. Voir aussi, Hocquart, 25 octobre 1730, C11A, 53: 200$200 \mathrm{v}$. Il est à noter que la saisie a été effectuée par le frère de Lanoullier, Jean-Eustache Lanoullier de Boisclerc, contrôleur de la Marine.

70 Dupuy, 27 octobre 1727, C11A, 49: 418-418v.

71 Hocquart, 17 octobre 1730, C11A, 53: 136v, 244.

72 Hocquart, ler octobre 1734, C11A, 62: 9-11. 
commissaire de la Marine, 177 livres; de Berey, le sous-commis même de Lanoullier, 1500 livres; et Berthier, chirurgien major, 637 livres ${ }^{73}$.

Enfin, il faut mentionner un dernier problème qui compliquait la tenue des comptes de la caisse. À cause du manque d'espèces qui sévissait au Canada, des lettres de change et des billets du commis devaient souvent tenir lieu de monnaie courante ${ }^{74}$. Ces papiers furent retirés en 1729, au moment de la réintroduction de la monnaie de cartes. Ils avaient jusque-là procuré certains avantages, surtout au niveau du commerce interne, mais aussi suscité des embarras. Dupuy, en 1727, puis Hocquart en 1730 s'étaient plaints qu'il était difficile d'arrêter les comptes à cause de la quantité de papiers qui circulait dans la colonie ${ }^{75}$.

Il n'est donc pas étonnant que la situation de Lanoullier soit devenue intenable. Il avait péché par imprudence en consentant de trop grandes avances aux officiers. Le temps qui s'écoulait entre l'arrivée et le départ du vaisseau du Roi, porteur des états financiers de France, était insuffisant pour lui permettre de régler sa comptabilité $^{76}$. Comme une partie des fonds destinés au Canada était déboursée dans les ports de France, il y avait une discordance presque constante entre les chiffres du commis et ceux des trésoriers généraux ${ }^{77}$. Enfin, le conflit violent qui éclata entre Dupuy et le gouverneur Beauharnois ne pouvait que nuire au bon fonctionnement des finances coloniales ${ }^{78}$. Assailli de toutes parts, Lanoullier semble peu à peu avoir perdu la maîtrise de sa caisse.

Dès 1729 , la situation était devenue si grave que Maurepas l'aborda comme premier point dans ses instructions au nouvel intendant, Gilles Hocquart:

73 Etat des recepisses du sieur Bauve», 15 octobre 1731, C11A, 55: 101-104.

74 Dupuy, 21 octobre 1726, C11A, 48: 293-293v. - Beauharnois et Hocquart, 25 octobre 1729, C11A, 51: 152-152v.

75 Dupuy, 20 octobre 1727, C11A, 49: 322v; répétée par Hocquart, 17 octobre 1730, C11A, 53: 132 ; et 19 octobre 1732, C11A, 58: 71-83. 48: $223-223 \mathrm{v}$.

76 Bégon, 26 octobre 1722, C11A, 44: 383v-384; Bégon, 12 octobre 1726, C11A,

77 Bégon, 2 novembre 1724, CIIA, 46: 163-163v; Maurepas à Dupuy, 24 mai 1728, CIIA, 50: 538-540. - Hocquart, 25 octobre 1729, CIIA, 51: 221-223 ; Hocquart, 15 octobre 1731, CIIA, 55: 159-164 (copie 194-202v).

78 D'Aigremont s'est plaint de Dupuy à cause de son refus de signer les comptes de 1725, CIIA, 50: 162-164v; D'aigremont, 8 novembre 1728, CIIA, 50: 257-269. 
Sa Majesté ayant rappelé au mois de mai de l'année dernière le sieur Dupuy intendant de la Nouvelle-France et étant informée que les affaires concernant les finances dans la colonie n'y sont point en règle, que les comptes des dépenses y sont en souffrance depuis 4 années et ne sont point arrêtés... sa Majesté s'est déterminée d'envoyer le sieur Hocquart en qualité d'ordonnateur ${ }^{79}$.

À l'égard de la conduite à tenir envers Lanoullier, les instructions ministérielles étaient sans équivoque:

Si le sieur Hocquart s'aperçoit que le sieur de Lanoullier ait diverti les fonds des recettes extraordinaires ou qu'il ait entré les dépenses contenues dans les bordereaux qui lui sont remis pour employer à son profit les lettres de change qui en sont provenues il s'assurera sa personne et prendra les plus justes mesures pour qu'il ne puisse pas s'évader et pour la sûreté des effets qu'il aura diverti soit pour les employer à son profit ou autrement ${ }^{80}$.

Ce ne fut qu'au mois de septembre 1730 que Hocquart s'occupa finalement des affaires de Lanoullier, quand il découvrit un vide dans le bordereau des dépenses et recettes de 1728. Le 5 septembre, il le destitua, le fit arrêter et ordonna l'apposition des scellés sur ses effets ${ }^{81}$. Le 15 du même mois, le contrôleur de la Marine, Jean Victor Varin de la Marre, déclara à l'intendant qu'il avait constaté un débit dans la caisse de Lanoullier de 301404 livres pour les années 1728 et 1729 et demanda la saisie desdits effets, pour assurer la sûreté des fonds du Roi ${ }^{82}$. Lanoullier, de son côté, présentait un état des sommes qui lui étaient dues par des particuliers en Nouvelle-France et dans la métropole, qui s'élevaient à 304269 livres. Cependant, selon Varin, ce relevé était "composé en partie de différents billets et reconnaissances des débiteurs dudit sieur la Noullier qui n'ont aucun rapport aux fonds du Roi qui ont été remis entre ses mains et dont sa Majesté ne doit être garente». Ainsi, Hocquart ordonna l'inventaire et la saisie des effets déjà mis sous scellés ${ }^{83}$. Cette partie de

79 «Mémoire du Roi pour servir d'instruction au sieur Hocquart», 22 mars 1729, B, 53: 471.

80 «Mémoire du Roi pour servir d'instruction au sieur Hocquart,» 19 avril 1729, B, 53: 510 .

81 CIIA, 53: 130-133v.

82 Varin à Hocquart, 15 septembre 1730, CIIA, 53: 246-247; Hocquart, 17 octobre 1730, CIIA, 53: 134v-135.

83 Ordonnance de Hocquart, 15 septembre 1730, CIIA, 53: 135. 
l'état de compte de Lanoullier déclarée non valable était composée d'avances aux officiers, mais l'état comprenait également des créances officielles faites dans le cadre des finances royales. On y retrouve des récépissés de Bauve, alors qu'il agissait comme commis; 57000 livres non remises par le garde-magasin Foucault pour les ventes de 1728; des reprises sur les trésoriers généraux, etc ${ }^{84}$.

Malgré la découverte de cet imposant débit dans les comptes de Lanoullier, Hocquart n'était pas convaincu que le commis avait agi avec malhonnêteté. Au procès-verbal de l'affaire, il joignit l'explication suivante:

Je ne dois point oublier, Monseigneur, de vous dire ce qui peut être en faveur du sieur Lanoullier. Le dérangement de sa caisse ne vient en partie que de la facilité qu'il a eue d'avancer aux officiers leurs appointements ${ }^{85}$.

Il insista particulièrement sur les avances faites à l'ordre de Bégon et Dupuy. Comme ces deux intendants avaient usé de leur pouvoir d'ordonnance pour les obtenir, ces sommes ne pouvaient être considérées comme des prêts personnels faits sur l'initiative de Lanoullier. L'année suivante, Maurepas lui-même exprimait des sentiments à peu près analogues. Le commis, selon lui, s'était attiré ses embarras "plus par l'envie de faire plaisir que par mauvaise foi» ${ }^{86}$. Ces opinions des hautes instances administratives expliquent pourquoi la punition infligée à Lanoullier ne fut pas très sévère. Il fut gardé en prison à peine deux ou trois jours, puis reconduit chez lui où il demeura en résidence surveillée. De plus, en dépit du caractère apparemment personnel des créances, Hocquart promit de faciliter «autant qu'il dépendra de [lui] les recouvrements à faire sur les officiers et sur les différents particuliers qui sont débiteurs dudit sieur Lanoullier ${ }^{87}$

Protégé par l'intendant, Lanoullier parvint graduellement à recouvrer les fonds qui lui étaient dus. En janvier 1731, Hocquart rapporta que l'ancien commis avait déjà récupéré environ 20 mille livres.

84 Ces non-valeurs furent justifiées par Hocquart, le 17 octobre 1730, CIIA, 53: 134-134v. Voir aussi le procès-verbal, CIIA, $53: 244-244 v$.

85 Hocquart, 17 octobre 1730, CIIA, 53: 138v-139.

86 Maurepas à Hocquart 10 avril 1731, CIIA, 56: 25-25v.

87 Hocquart, 17 octobre 1730, CIIA, 53: 136. Néanmoins, en 1732, quand Lanoullier a demandé de faire charger le contrôleur de la Marine de la poursuite au nom du Roi des 14856 livres de non-valeurs restantes, Hocquart refusa. Hocquart, 19 octobre $1732,58: 71-83$. 
Puis, en octobre de la même année, malgré une augmentation de 'sa dette de 13250 livres, Lanoullier, au moyen de nouveaux recouvrements s'élevant à 265130 livres, réduisit son débit à 49524 livres ${ }^{88}$. Il promit de rembourser cette dernière somme au moyen d'un paiement de 35000 livres au mois d'octobre 1732, paiement pour lequel Pierre de Lestage, marchand de Montréal, se porta garant ${ }^{89}$. Pour ce qui est des 14524 livres restantes, Hocquart accepta comme garantie la maison de Lanoullier à Québec et une caution additionnelle de 20000 livres en France. Cependant, en octobre 1732, Lanoullier n'avait réduit son débit qu'à 42206 livres et il promit alors de payer 24000 livres provenant de la vente des marchandises au mois de décembre. De plus, il remit à Hocquart un mémoire de créances encore dues s'élevant à 14856 livres qu'il était en train de recouvrer et grâce auxquelles il ne serait plus redevable que de 3350 livres ${ }^{90}$. Toutefois, même en 1750, Lanoullier n'avait pas encore acquitté sa dette à la Marine et cherchait toujours à recouvrer des sommes de ses débiteurs ${ }^{91}$.

La faillite de Nicolas Lanoullier fut causée par des facteurs d'ordre financier, institutionnel et social. Hocquart en avait relevé certains au cours de son enquête, notamment l'habitude qu'avait Lanoullier d'utiliser indifféremment les deniers royaux dont il avait la charge pour des affaires publiques et des affaires privées. Pour corriger cette situation, il lui semblait souhaitable que le commis ne s'occupât que des fonctions de la Marine et ne se mêlât pas du commerce de la colonie. L'intendant croyait que la modicité de ses appointements porterait toujours le commis à faire du commerce; il fallait donc commencer par les augmenter.

Comment, avec 1200 livres qu'ils (les trésoriers généraux) donnaient au sieur Lanoullier peuvent ils entendre que telle service soit bien faite. Il n'est pas possible qu'un homme aussi malpayé ne cherche et ne trouve dans sa caisse des ressources. Il est encore nécessaire qu'ils lui interdisaient tout commerce ${ }^{92}$.

88 Hocquart, 15 janvier 1731, CIIA, 55: 6v; «État des récépissés du sieur Bauve», 15 octobre 1731, CIIA, 55: 101-104.

89 Billet de Lanoullier et un cautionnement de Lestage, 12 octobre 1731, CIIA, $55: 282$.

90 Hocquart, 15 octobre 1731, CIIA, 55: 159; Hocquart, 19 octobre 1732, CIIA, 58: 80-82.

91 Liste de non-valeurs de Lanoullier, 23 octobre 1750, C11A, 61: 274-275.

92 Hocquart, 16 octobre 1730, CIIA, 53: 55v-56. 
Toujours selon Hocquart, Lanoullier avait aussi été victime des méthodes utilisées par les intendants pour ordonner les fonds. Parlant de Lanoullier il dit:

Je sais qu'il a souvent eu des ordres verbaux qu'il n'a pu se dispenser d'éxécuter et qui l'ont fait tomber dans des doubles emplois auxquels j'aurai égard si vous le trouvez bon ${ }^{93}$.

Évidemment, cette situation ne s'était guère améliorée depuis 1720 , quand l'ancien commis Jean Petit avait présenté des griefs semblables $^{94}$.

Hocquart prit donc énergiquement parti pour Lanoullier, notamment contre les trésoriers généraux qu'il accusa de l'avoir maltraité ${ }^{95}$. Il prétendit même que ceux-ci lui devaient une compensation:

Messieurs les trésoriers généraux lui ont refusé constamment de lui allouer dans ses comptes de clerc à maître plus de 1200 livres pour ses appointements. Il n'a pu faire leur service pour une somme si modique. Le détail d'un trésorier en ce pays exige presqu'autant de travail que l'employé de trésoriers dans un port, ainsi je vous supplie, Monseigneur, d'engager les trésoriers généraux à traiter le sieur Lanoullier plus favorablement. Ils ont reconnu cette demande en accordant au sieur Taschereau les appointements de 2000 livres $^{96}$.

Lanoullier continua de bénéficier des grâces de Hocquart et du gouverneur Beauharnois, sous forme de gratifications, de privilèges, etc. jusqu'à sa mort en 1756. En 1733, Hocquart expliqua ce traitement de faveur dont jouissait l'ancien commis en déclarant que le désordre de ses affaires venait "peut être moins de sa faute que de celle des différentes circonstances des temps ${ }^{97}$.

\section{Conclusion - Un cas typique}

La charge de commis des trésoriers généraux de la Marine fut très importante dans la gestion des affaires financières de la

93 Hocquart, 17 octobre 1730, CIIA, 53: 135v.

94 Conseil à Bégon, 20 avril 1720, CIIA, 41: 258v-259.

95 Hocquart, 25 octobre 1729, CIIA, 51: 222v-223; Hocquart, 15 octobre 1731, CIIA, 55: 162.

96 Hocquart, 8 octobre 1733, CIIA, 60: 67-67v.

97 Hocquart, 8 octobre 1733, CIIA, 60: 62-62v. Voir aussi, Beauharnois, 10 octobre 1733, CIIA, 59: 41-41v; Hocquart, 8 octobre 1733, CIIA, 60: 62-67v. 
Nouvelle-France. L'exercice de ces tâches constituait un rouage absolument essentiel et conférait aux titulaires une importance reconnue à l'intérieur de l'administration coloniale. Cette charge, toutefois, comme celle du trésorier général en France, fut une affaire privée, plus près par sa nature d'une entreprise bancaire que d'un office royal. Malgré la surveillance qu'exerçait périodiquement l'intendant, le commissaire de la Marine et le contrôleur de la Marine, il demeure que ces fonctions ne relevaient pas de la juridiction propre des administrateurs royaux. C'est donc, par exemple, avec l'optique d'un entrepreneur plutôt que d'un administrateur que Nicolas Lanoullier de Boisclerc accéda au poste en 1720 et l'assuma jusqu'à 1730. Il croyait faire une bonne affaire quand il l'accepta et ne cessa point d'en chercher d'autres ${ }^{98}$. Ainsi, au cœur même de l'appareil financier de l'administration coloniale se trouvait un poste que l'on peut qualifier d' "entrepreneurship», ce qui ne manqua pas d'entrâ̂ner des conséquences fâcheuses pour Lanoullier durant presque tout son exercice.

Sur le plan politique et social, ce poste fit partie d'un système de relätions personnelles qui dépassait le cadre des charges officielles. Séparé des administrateurs royaux par son statut de commis des trésoriers, le titulaire fut néanmoins lié à l'intendant et à d'autres officiers de la Marine pour les dépenses et les vérifications officielles. Puis s'ajoutant à ces éléments fonctionnels, on retrouve les liens que créent les avances financières aux officiers, les alliances au Conseil Supérieur où siégeaient les commis, et même, parfois, des liens familiaux, qui se réunissent pour devenir un réseau étendu d'intérêt commun. La carrière de Lanoullier fait voir nettement les avantages d'une telle situation. Deux intendants et le commissaire de la Marine lui devaient des sommes importantes avancées des fonds de la Marine et le contrôleur, jusqu'à 1729, Jean-Eustache Lanoullier de Boisclerc, était son demi-frère. C'est ce qui lui permettait, semble-t-il, de continuer l'exercice de ses fonctions malgré les soupçons et malgré les plaintes portées contre sa gestion comme commis dès les premières années.

En fin de compte, la fonction de commis des trésoriers généraux de la Marine subit les effets d'un système financier colonial

98 Par exemple; Lanoullier fut parmi les particuliers intéressés au bail du poste de Témiscamingue en 1725 , et lui-même a fait une offre, trop minime toutefois pour gagner la préférence, CIIA, 48: 235-238. 
troublé tant au chapitre des fonds qu'à celui de l'organisation, et aussi des influences fâcheuses liées à l'administration même de la Nouvelle-France. À cet égard, la carrière de Nicolas Lanoullier de Boisclerc fut typique de celle des commis et, comme ses prédécesseurs, Jean Petit et Georges-Regnard Duplessis et son successeur Thomas-Jacques Taschereau, il se trouva en déficit à la fin de son exercice. 